\title{
Informed consent document in gastrointestinal endoscopy: understanding and acceptance by patients
}

\author{
J. J. Vila, F. J. Jiménez, M. Iñarrairaegui, C. Prieto, O. Nantes and F. Borda \\ Department of Gastroenterology. Hospital de Navarra. Pamplona, Spain
}

\begin{abstract}
Objective: we wanted to know if patients read and understand the informed consent (IC) document used for endoscopic procedures, and to evaluate the readability of IC.

Method: during two months we gave patients studied in our endoscopy unit an anonymous questionnaire with different items concerning reading degree, knowledge of the technique, complications, sedation used, and information received. We evaluated IC readability using the Flesch index.

Results: 309 patients were included (mean age: 53 years, $55 \%$ males, $86 \%$ outpatients, $50 \%$ with basic education); $85 \%$ of patients read the IC, 96\% considered they understood the exploration technique, $22 \%$ were not aware of severe complications, and $82 \%$ knew which kind of sedation would be used; $88 \%$ of patients received additional information from their doctors. Outpatients read the IC in a greater percentage versus inpatients $(p<$ 0.05 ); patients with only basic education tended to ignore the possibility of complications $(p<0.05)$. Doctors gave more information to rural patients $(p=0.08)$, offered better information about complications to urban patients $(p=0.09)$, and offered more information on other diagnostic procedures to patients older than 50 years $(p<0.05)$. With the Flesch index we found that gastroscopy and colonoscopy ICs had a "standard" level of readability, while ERCP ICs were more complex.

Conclusions: the majority of our patients read and understands the IC. Doctors adapt information to patient characteristics. Our IC documents have an acceptable level of readability, but given that $50 \%$ of our patients have only a basic educational status, we should attempt to provide an easier IC document.
\end{abstract}

Key words: Informed consent. Endoscopy. Comprehension. Language tests.
Vila JJ, Jiménez FJ, Iñarrairaegui $M$, Prieto $C$, Nantes $O$, Borda F. Informed consent document in gastrointestinal endoscopy: understanding and acceptance by patients. Rev Esp Enferm Dig 2006; 98: 101-111.

\section{INTRODUCTION}

In the last few decades the patient-physician relationship has gradually evolved to a new relationship in which the patient's right of autonomy is recognized. Due to this autonomy, patients have the capacity to choose the best option for them and play an active role in the diagnostic and therapeutic approach of their own illness. For this reason they need to have adequate information about the proposed diagnostic procedures, as well as their alternatives. Nowadays it is considered that a system based on a single principle (beneficence or autonomy) is not a good situation, and we are tending towards a balanced relationship called enhanced autonomy (1), in which physicians as well as patients play an active role.

As a consequence of this change in the patient-physician relationship, the concept of informed consent (IC) appears as the documental basis verifying that a patient has received suitable information from his/her doctor, and understands said information.

Physicians must offer suitable information within a progressive process $(2,3)$, in which they must provide the information that patients want to know, but also information patients must know in order to preserve their autonomy without malefficiency (4). This way, patients can make a decision based on real and synthetic information.

Thus, physicians must not play a passive role and limit themselves to the offering of written information following patient wishes. They have to complement this written information with oral information, including their expert 
opinion and advice. Physicians must not use coercion but persuasion.

The signed form must be only a warranty that the patient has not accepted a long list of possible risks almost coercively, but has understood a proposal with its inherent risks-to-benefits balance (5).

Although providing patients with an IC document is a routine activity in clinical practice, the philosophy of IC has not been well understood by both patients and physicians.

Physicians may see it as a legal imposition to avoid litigation or as a detriment in their decision-making capacity. Patients may feel it is a way to share responsibility not for the decision but for complications, thus releasing physicians from this responsibility. Moreover, a patient may become a health system user with the right to obtain results, and go to court should these fail to materialize (6). Some authors think that patients adopt this attitude when IC forms are their only source of information (7).

In our country, the IC process, with the right of patients and their families to receive adequate information regarding their diagnosis, prognosis and treatment is ethically, legally, and deontologically supported (8).

In our endoscopy unit we use different IC forms adapted to the various endoscopic explorations. These IC forms were developed in 1996 by an IC Expert Committee for Navarre, which had the mission of progressively developing and implementing IC forms in hospitals in this region (9).

The aims of this study included an assessment of endoscopic IC acceptance and understanding by our patients. We also wanted to analyze the readability of IC forms in order to assess how these texts are understood according to the social and cultural characteristics of our patients.

\section{PATIENTS AND METHODS}

We developed a questionnaire in which we recorded the following epidemiological data: age, gender, patient source (inpatient or outpatient), educational level (from basic school to university), and place of residency (rural or urban environment). According to the rules of the Spanish Statistical National Institute, we considered as rural villages with less than 10.000 inhabitants, and urban those with more than 10.000 inhabitants.

In the questionnaire, besides these epidemiological data we asked patients:

1. Whether they had read the IC document.

2. Whether they had accepted the endoscopic exploration. Patients who refused were asked whether the information included in the IC document was determinant for their decision.

3. Whether, once the endoscopic exploration had been performed, they considered that they knew enough about the endoscopic technique, taking into account the information given in the IC document.
4. Whether they were aware of the possibility that even severe complications could happen during the endoscopic procedure.

5. The knowledge they had about the kind of sedation that would be administered during the exploration.

6 . Whether they considered that the information given in the IC document was quantitatively appropriate, as well as easily understood.

7. Whether they considered the IC useless or useful for patients, or thought that its main objective was to release doctors from responsibility.

8. Whether they had received oral information from their doctors. Whether they thought that such information was enough concerning the endoscopic technique, its complications, and alternative explorations.

9. Their subjective opinion about the information given by doctors.

10. Whether they would agree to sign one general IC document that would permit physicians to perform any exploration they considered appropriate by just telling their patients what they had decided to do, or if, on the contrary, they wanted to receive detailed information about each exploration proposed.

The IC document was handed over by the clinician to patients at the time of consultation, as usual at least 24 hours before the procedure. In contrast, the questionnaire was provided by the endoscopist in the endoscopy unit once the exploration had been performed. The questionnaire was completed anonymously by patients outside the endoscopy unit, with no further contact with the endoscopy unit personnel. It was then put by patients inside a letterbox before leaving our ward.

Patients who did not accept the endoscopic exploration were also asked to anonymously fill in the questionnaire once the consultation was over, outside the doctor's office.

We excluded urgent explorations and patients with a clinical situation as poor as to preclude questionnaire completion.

To analyze the readability of informed consent forms, we used the Flesch index test as adapted to the Spanish language. We applied this test to gastroscopy, colonoscopy, and endoscopic retrograde cholangiopancreatography (ERCP) IC forms. This index is calculated using the following formula:

$$
\mathrm{FL}=206.84-0.6 \mathrm{P}-1.02 \mathrm{~F}
$$

where $\mathrm{P}$ is the average number of syllables for each 100 words, and $\mathrm{F}$ is the average number of sentences for each 100 words.

Using this formula, we obtain a value within a scale from 10 to 100, where lower scores mean worse readability and higher scores correspond to better readability (Table I).

The statistical analysis was performed using the SPSS v 11.5 program. We applied Student's t, Chi-Square, and 
Table I. Flesch-Kincaid scoring system

\begin{tabular}{cc}
\hline Level & Reading difficulty \\
\hline $90-100$ & Very easy \\
$80-90$ & Easy \\
$70-80$ & Fairly easy \\
$60-70$ & Standard \\
$50-60$ & Rather difficult \\
$30-50$ & Difficult \\
$0-30$ & Very difficult \\
\hline
\end{tabular}

Fischer's exact tests when appropriate. Differences between variables were considered statistically significant when the $p$ value was below 0.05 . We also include confidence intervals (CI) with a confidence level of $95 \%$.

\section{RESULTS}

We asked 339 consecutive patients seen in our endoscopy unit for their participation in the study; 309 of them $(90 \%)$ agreed to complete the questionnaire.

Concerning patient source, $85.8 \%$ of patients were outpatients and $63.8 \%$ of them lived in an urban environment. Sex distribution was $55.1 \%$ males and $44.9 \%$ females. Mean age was $53.3 \pm 17.2$ years, ranging between 15 and 90 years. Almost half of the patients $(49.7 \%)$ had only basic education, $28.9 \%$ had pre-university education and $21.4 \%$ had university studies; $79 \%$ of patients were referred by a gastroenterologist, while $11 \%$ of patients came from other specialized offices and primary care.

Almost all patients included (98.7\%) accepted the endoscopic exploration when it was proposed by their doctors. Four patients $(1.3 \%)$ did not agree, and only one of these patients said that the information provided in the IC document was the main reason for his decision.

With regard to reading extent, $84.9 \%(\mathrm{CI}=79.2$ $87.7 \%$ ) of patients read the IC document. More outpatients read the IC document versus inpatients (86.4 vs. $73.8 \% ; \mathrm{p}=0.035)$.

When we compared educational level with age we found that $66 \%$ of patients older than 50 years had only primary studies, while only $28 \%$ of younger patients had that level of education $(\mathrm{p}=0.001)$.

Taking into account the information given in the IC document, and once the endoscopic procedure had been performed, $79.9 \%$ ( $\mathrm{CI}=75-84.2 \%$ ) of patients considered they understood reasonably well the exploration technique, while $4.3 \%$ ( $\mathrm{CI}=1.7-6.2 \%)$ considered they did not, and $16.5 \%$ did not answer this question.

In all, $67 \%(\mathrm{CI}=61.4-72.2 \%)$ of patients who had read the IC document were aware that even severe complications could happen during the endoscopic exploration, $15.2 \%(\mathrm{CI}=11.3-19.7 \%)$ were not, and $17.8 \% \mathrm{did}$ not answer. We noticed a trend to ignore this possibility in patients with only primary education $(\mathrm{p}=0.052)$.
Furthermore, $68.6 \%(\mathrm{CI}=63.1-73.7 \%)$ of patients knew the kind of anesthesia they would receive during the endoscopic procedure, and we noticed a trend in outpatients to be more aware of this issue $(p=0.062)$.

Regarding the subjective opinion of patients about the amount of information given in the IC document, $77.3 \%$ $(\mathrm{CI}=72.2-81.8 \%)$ of patients considered it adequate, while $5.2 \%(\mathrm{CI}=2.9-8.2 \%)$ considered it excessive, and $3.2 \%(\mathrm{CI}=1.5-5.8 \%)$ found it insufficient. The question was not answered by $14.2 \%$ of patients.

Regarding the subjective understanding of information in the IC document, 71.8\% ( $\mathrm{CI}=66.4-76.7 \%)$ found it adequate, $9.1 \%(\mathrm{CI}=6.1-12.8 \%)$ found it too complex, and $2.6 \%(\mathrm{CI}=1.1-5 \%)$ felt it was too simple. This question was not answered by $16.5 \%$ of patients.

When we related these subjective opinions to age and educational level, we noticed in patients older than 50 years a trend to consider information amount as insufficient $(\mathrm{p}=0.06)$, and understandability as too complex ( $\mathrm{p}$ $=0.083$ ). The latter opinion was shared by patients with only primary education $(\mathrm{p}=0.032)$.

Also, $64.3 \%(\mathrm{CI}=58.3-70 \%)$ of patients thought that the IC document was really a useful document for patients, $3.7 \%(\mathrm{CI}=1.7-6.6 \%)$ considered it useless, and $32 \%(\mathrm{CI}=26.4-37.8 \%)$ thought that it is mainly a tool to release doctors from responsibility.

With regard to the role of physicians, $87.6 \%(\mathrm{CI}=83$ $91.2 \%$ ) of patients had received oral information from their doctors, while $12.4 \%$ ( $\mathrm{CI}=8.7-16.9 \%)$ had not. We found in doctors a trend to give more information to patients with only primary education $(90.8 v s .83 .3 \%$; $\mathrm{p}=$ $0.07)$, and to those who live in rural environments $(92.2$ vs. $84.27 \% ; \mathrm{p}=0.072$ ).

In addition, $93 \%$ of patients $(\mathrm{CI}=89.1-95.7 \%)$ who had received information from their doctors were informed about the exploration technique, $63.6 \%$ (CI $=$ $57.3-69.5 \%$ ) were briefed about potential complications, and $74.4 \%(\mathrm{CI}=66.8-80.9 \%)$ were educated on alternative explorations to endoscopic procedures.

In analyzing these data we found that doctors give more information about the alternatives to endoscopic exploration to patients older than 50 years (78.5 vs. $63.3 \%$; $\mathrm{p}=0.044$ ); with regard to potential complications, doctors tend to give more information to patients who live in an urban environment (68 vs. $56.4 \% ; \mathrm{p}=0.079)$.

Thus, $78 \%$ (CI $=72.9-82.4 \%)$ of patients felt that information received from their doctors was adequate, $1.6 \%(\mathrm{CI}=0.5-3.7 \%)$ considered it excessive, and 9.1\% $(\mathrm{CI}=6.1-12.8 \%)$ thought of it as insufficient. Approximately, eleven percent $(11,3 \%)$ of patients refused to evaluate this point.

Finally, 38.5\% (CI $=32.6-44.6 \%)$ of patients said that they would rather sign only one global IC document, with no need to sign a distinct form for each exploration proposed within their diagnostic approach. The rest of patients $(61.5 \%$; $\mathrm{CI}=55.3-67.3 \%)$ preferred to be informed 
for each individual procedure and to sign individual forms. We found a significant statistical difference concerning sex, in such a manner that women are more reluctant to sign a global form and would rather be informed in detail and sign a separate form for each exploration ( $p$ $=0.018$ ).

The results of the Flesch index test for the gastroscopy and colonoscopy forms were 67.3 and 64.5, respectively, both of them corresponding to a standard level of readability. On the other hand, the result for the ERCP form was 55.56, corresponding to a fairly difficult level of readability.

\section{DISCUSSION}

To offer correct and suitable information is not always easy for physicians, especially considering that it is often really difficult to guess what patients want to know. An interesting study has proved that there are important differences between what patients and solicitors consider appropriate concerning the right moment to provide information, the number of times that information should be given, and the minimum level of risk for complications that patients should be warned of (10).

The majority of patients in our study read the IC document $(84.9 \%)$. This percentage is lower than that reported in other previous studies made in other countries, but higher than the percentage reported in a study made a few years ago in our country by Parera et al. (6). The latter study, which we consider most comparable to ours since epidemiological characteristics and patient origin are similar, reported somewhat differing results when compared to our study.

First of all there is an important difference concerning study methodology. In contrast to us and other authors (11), Parera et al. gave patients the questionnaire before the exploration was made. In this way patients read the questions before the procedure, this allowing them to consult the IC document if they had not read it before. This may improve final results regarding understanding, especially information concerning the exploration technique.

On the other hand this type of studies are really quality and satisfaction studies in which it is recommended that patients be questioned about the studied subject when they are not going to have any more contact with the evaluated system. It is even advisable that questions be asked by phone or mail (12). We wanted to minimize the influence of a patient's aspiration to please the system that is diagnosing and treating his disease by giving the questionnaire after the procedure and having it filled in anonymously by the patient alone, with no more contact with the endoscopy unit personnel.

Secondly, we disagree with Parera et al., as well as with other authors (13), when they declare that patients demand increasingly less information. In our study, read- ing extent and the percentage of patients who receive oral information from their doctors are significantly greater than those in the study by Parera et al., which was made in the same country and with a similar population (14).

We excluded urgent explorations when taking into account that in such situation IC is neither autonomous nor informed (15), two key points for the validity of the IC process. On the contrary, we included patients who rejected the endoscopic exploration in order to evaluate the influence that the fact of reading the IC form may have on patient decision-making. The very few patients who rejected the exploration prevented us from reaching more conclusions in this respect.

According with Parera et al., we found a relationship between degree of understanding and educational level: patients older than 50 years considered the text in the IC form too complex and the amount of information too short. These two questions are probably interrelated, as patients older than 50 years have a poor understanding of the text and therefore receive less information.

We want to emphasize that $15.2 \%$ of patients who read the IC document ignored that even severe complications could happen during the endoscopic exploration, while this subject is clearly explained in the IC document. We found in patients with only primary education a trend to ignore the risk of complications, probably related to the difficulties of these patients in understanding the IC text.

Thus physicians should pay special attention to these patients when providing oral information. In this respect, physicians give more information to patients with only primary education and patients who live in a rural environment. This may be due to the fact that physicians are conscious of the difficulties that these patients have in understanding the information provided, or the fact that these patients ask for more information.

An adaptation of the information that doctors offer to patients is also seen in the population older than 50 years, who receive more information on the alternatives to endoscopic exploration from doctors.

We take this as a sign of persuasion by the doctor, who may consider it adequate to perform other diagnostic explorations in older patients and in patients with more systemic conditions. In contrast, urban patients receive more information about possible complications.

Outpatients showed a greater reading extent and a better knowledge of the kind of sedation they were going to receive. This could be related to many factors, such as the probable better health condition of outpatients versus inpatients, as well as a bigger lapse of time until the exploration is made allowing a repeated and detailed reading of the IC form, which leads to better comprehension. In addition, inpatients are permanently in touch with doctors and nurses, who play a very important role in the IC process (4).

The great majority of our patients came from a gastroenterology clinic or ward. This could be a selection 
bias. The rest of patients came from other specialty offices or wards, or directly from primary care physicians with an open access system. Although this kind of open access system has been shown to influence the IC process (16), in our study we noticed no differences in this sense; however, we cannot rule out that this is due to the small amount of patients studied by this system (the statistical power of this analysis is under $15 \%$ ).

In our study only $32 \%$ of patients considered that the IC document is basically a tool to release doctors from responsibility, considerably lower than the percentages reported by other similar studies about the same subject before $(6,11,14)$. This and all the issues we have discussed before (greater reading extent, greater amount of oral information given by doctors, adaptation of this information to the characteristics of patients) may reflect that the philosophy of the IC process and the balanced situation we mentioned before in the introduction are being assimilated by both patients and physicians. This could be the main reason behind the differences between our study and other studies (6).

With regard to the readability of IC forms, there have been recent reports on readability analyses for IC forms as used for clinical trials. The results of these studies show that the readability of these IC forms is really poor $(17,18)$. We wanted to evaluate if this held also true for the IC forms we use in our endoscopy unit.

For our readability analysis we used the Flesch-Kincaid index as adapted to the Spanish language (19). This test has been recently used in our country for readability and comprehension analyses of medical information (20).

The results of our study show that the readability of the IC forms for gastroscopy and colonoscopy is standard, adapted to the reading capacity of a standard thirteen-year-old schoolboy. The text in the ERCP IC form is more demanding to read, and is adapted to a pre-university reading level (20).

Readability value is only an orientation on the comprehension patients may have of a text. The comprehension of a text depends on readability but also on other various factors such as cultural level, level of health knowledge, and motivation degree (18). Furthermore, readability might be influenced by the kind of letters used, the exposition and extent of the text (21).

However, the results of our study are an indication that we must make an extra effort to improve readability in the texts we use in our endoscopic IC forms, specially considering that older patients may not have the reading and comprehension skills necessary to successfully understand information in a health environment (22). In our own study we found that older patients and patients with only primary education have the greatest difficulties in understanding IC forms. In addition, the readability and comprehension skills that patients have are usually lower than their educational level. For this reason, the United States Health Department recommends that documents used for educational purposes should be adapted to the readability level of an elevenyear-old child (20).

In conclusion, as has been said before, we must make our IC forms more legible, improving this way the reading a comprehension level of our patients older than 50 years or with only primary education. These are the patients in our environment who have more difficulties in understanding IC forms, and in receiving adequate information.

Finally, we agree with Rex et al. that we should add to IC forms information on the existing possibility that false-negative explorations may happen $(23,24)$. This is important not only to favor the IC process but also to decrease an important source of legal disputes in our specialty (25).

\section{REFERENCES}

1. Quill TE, Brody H. Physician recommendations and patient autonomy. Finding a balance between physician power and patient choice. Ann Intern Med 1996; 125: 763.

2. Alleger M, Coos E. Consentimiento Informado. Gastroenterol Hepatol Continuada 2003; 2: 89-54.

3. Garrido Sanjuán JA. Training in communication and informed consent in our system of medical resident: what information should and can give a resident? Med Clin (Barc) 1998; 111: 739-41.

4. Kanoti G. Ethics and medical-ethical decisions. Crit Care Clin 1986; 2: 3 .

5. Broggi Trías MA. Informed or uninformed consent? The risk of defensive medicine. Med Clin (Barc) 1999; 112: 95-6.

6. Parera de Cáceres A, González Asanza C, Melchén Fernández-Pacheco PL, et al. Survey of informed consent in a gastrointestinal endoscopy unit. Gastrenterol Hepatol 2000; 23: 317-21.

7. Acea Nebril B. Informed consent in gastrointestinal endoscopy. Who should inform? Gastroenterol Hepatol 2001; 24: 146.

8. Simón Lorda P, Júdez Gutiérrez J. Informed Consent. Med Clin (Barc) 2001; 117: 99-106.

9. Comité de Expertos en Consentimiento informado del Departamento de Salud/Gobierno de Navarra. Orden Foral 151/1996, de 18 de julio, del Consejero de Salud del Gobierno de Navarra. Informed Consent. An Sist Sanit Navar 1997; 20.

10. Mayberry MK, Mayberry JF. Towards better informed consent in endoscopy: a study of information and consent processes in gastroscopy and flexible sigmoidoscopy. Eur J Gastroenterol Hepatol 2001; 13: 1467-76.

11. Denis B, Bottlaender J, Goineau J, et al. Consentement éclairé en endoscopie digestive. Enquête d'opinion apures des malades. Gastroenterol Clin Biol 2002; 26: 675-9.

12. Shepherd HA, Bowman D, Hancock B, et al. Postal consent for upper gastrointestinal endoscopy. Gut 2000; 46: 37-9.

13. Ibáñez E. Un intento de explicar el porqué. JANO 1991; 40: 37-45.

14. Roque I, Hochain P, Merle V, et al. Evaluation de la qualité et de 1'impact de l'information transmise par les fiches d'endoscopie digestive elabores par les sociétes savantes. Gatroenterol Clin Biol 2003; 27: 17-21.

15. Williams BF, French JK, White HD. HERO-2 consent substudy investigators. Informed consent during the clinical emergency of acute myocardial infarction (HERO-2 consent substudy): a prospective observational study. Lancet 2003; 361: 918-22.

16. Eisen GM, Baron TH, Dominitz JA, et al. Standards of Practice Committee of the American Society for Gastrointestinal Endoscopy. Gastrointestinal Endoscopy 2002; 56: 793-5.

17. Gost J, Silvestre C, Ezpeleta P, et al. Evaluation of the clinical practice of informed consent in clinical trials. An Sist Sanit Navar 2003; 26: $35-42$. 
18. Ordovás Baines JP, López Briz E, Urbieta Sanz E, et al. Analysis of the written patient information form to obtain informed consent in clinical trials. Med Clin (Barc) 1999; 112: 90-4.

19. Fernández Huerta J. Medidas sencillas de lecturabilidad. Consigna 1959; 214: 29-32.

20. Blanco Pérez A, Gutiérrez Couto U. Legibilidad de las páginas web sobre salud dirigidas a pacientes y lectores de la población general. Rev Esp Salud Pública 2002; 76: 321-31.

21. Paasche-Orlow MK, Taylor HA, Brancati FL. Readability standards for informed-consent forms as compared with actual readability. $\mathrm{N}$ Engl J Med 2003; 348: 721-6.
22. Gazmararian JA, Baker DW, Williams MV, et al. Health literacy among medicare enrollees in a managed care organization. JAMA 1999; 281: 545-51.

23. Rex DK, Culter CS, Lemmel GT, et al. Colonoscopic miss rates of adenomas determined by back to back colonoscopies. Gastroenterology 1997; 112: 24-8.

24. Gerstenberger PD, Plumeri P. Malpractice claims in gastrointestinal endoscopy: An analysis of an insurance industry database. Gastrointestinal Endosc 1993; 39: 132-8.

25. Rex DK, Bond JH, Feld AD. Medical-Legal risks of incident cancers after clearing colonoscopy. Am J Gastroenterol 2001; 96: 952-7. 